\title{
Diversity in aerial root anatomy of Bulbophyllum (Orchidaceae) and its significance as source for subsidiary characters in species identification
}

\author{
Madhavi Singh ${ }^{1}$, Vimala Y. ${ }^{2}$, Lavania S. ${ }^{1 *} \&$ D. Verma ${ }^{3}$ \\ 1Department of Botany, University of Lucknow, Lucknow, Uttar Pradesh - 226007 , India \\ 2Department of Botany, C.C.S. University, Meerut, Uttar Pradesh - 250 004, India \\ 3Botanical Survey of India, Dehradun, Uttarakhand - 248 195, India \\ *E-mail: lavaniaseshu@yahoo.co.in
}

\begin{abstract}
The morphology and anatomy of aerial roots of twelve epiphytic species of Bulbophyllum Thouars were investigated to analyse the interspecific variation and their potential for species differentiation and identification. Anatomical features of various structures such as velamen tissue, exodermis, cortex, endodermis, stele, thickening pattern, the occurrence of raphides, crystals, druses in the cortical region and types of tracheoidal (water storage) cells were examined. Tilosomes were lamellate in half of the species studied, while in B. umbellatum Lindl. they were spongy. The exodermal cells showed uniform cell wall thickening all around cells in all the species analysed except in $B$. affine Wall. ex. Lindl. and B. striatum (Griff.) Rchb.f. where they were inverted U-shaped. Raphides, druses and a few geometrically shaped calcium oxalate crystals were found in the cortical cells, while they were absent in B. affine and B. umbellatum. A positive correlation was found between stele diameter with root area and cortex thickness, number of xylem strands with root diameter and velamen thickness, velamen thickness with root diameter and stele diameter as in root cross sections. The variation in exodermis thickening, ideoblast cells, crystal forms, presence or absence of chloroplast cells in cortex, tilosome type as observed in the present study may be useful as potential distinguishing features and additional characters for taxonomic identification of Bulbophyllum species.
\end{abstract}

Keywords: Druse, Epiphytic, Exodermis, Tilosomes, Tracheoidal cells, Velamen.

Received: 16.04.2021 ; Revised \& Accepted: 02.12.2021

Published Online: 31.12 .2021

\section{Introduction}

The family Orchidaceae Juss. is a highly evolved plant family represented by 736 recognised genera (Chase et al., 2015) and 25,000 species distributed throughout the world (except Antarctica) and showing a wide range of variation in its morphology and anatomy (Atwood, 1986; Dressler, 1993). Orchids are also known for their morphological and anatomical adaptations, which produce an array of unique characters for identification and classification (Pridgeon \& Stern, 1982; Aybeke et al., 2010; Fan et al., 2014).

Among various genera, the genus Bulbophyllum Thouars has a pantropical distribution, and includes about 2,200 species inhabiting diverse climates across the world (Pridgeon et al., 2014). Amongst them nearly 100 species are reported from India. In this genus, growth and survival of a plant is supported by a number of morphological and anatomical adaptations in the floral and vegetative parts including the roots (Micco \& Aronne, 2012). The aerial root anatomy of Bulbophyllam careyanum (Hook.) Spreng. was first investigated by Chatin (1856). Subsequently, Oudemans (1861) described the epidermal layer and its position in aerial roots of Bulbophyllum. Afterward, Leitgeb (1864) reported single-layered velamen in B. muscicola Rchb.f., and Meinecke (1894) in Bulbophyllinae Schltr. Dressler (1981) pointed out the presence of a white coloured sheath that enclosed the root in most orchids and called it 'velamen radicum'. Later 
Pridgeon (1987) defined velamen radicum as the outermost, spongy, single to multi-layered, thick walled, dead (at maturity) structure of aerial roots. Porembski and Barthlott (1988) also reported single-layered velamen in Bulbophyllum. Pridgeon et al. (1983) described the morphological types of tilosomes, protrusions or wall ingrowths in the form of branched lignified structures on the inner periclinal wall of the velamen (Stern, 2014).

Aybeke (2012) studied the taxonomic importance of anatomical characters of leaf, rhizome and root of various species of orchids for the identification of some taxa of the subfamilies Orchidoideae and Epidendroideae. Epiphytic plants grow on substrata like rocks, tree bark (at different heights on tall trees of different species), and their roots emerge from rhizomes that are exposed to light having intermittent access to water and minerals. Therefore, the aerial root morphology is entirely different from that of underground roots of orchids (Thangavelu \& Kowsalya, 2017). As such, aerial roots of orchids have been shown to provide valuable anatomical characters for species diagnosis in taxonomy (Singh, 1986; Piazza et al., 2015). The present study was conducted to elucidate anatomical features of the aerial roots to identify the supplementary diagnostic characters that could complement the application of leaf epidermal characters in the context of species identification (Singh et al., 2020).

\section{Materials and Methods}

\section{Sample collection}

Living plants of twelve epiphytic taxa of the genus Bulbophyllum, namely, B. affine Wall. ex. Lindl., B. ambrosia (Hance) Schltr., B. bisetum Lindl., B. cauliflorum Hook.f., B. cherrapunjeense Barbhuiya \& D.Verma, B. gymnopus Hook.f., B. leopardinum (Wall.) Lindl. ex. Wall., B. pteroglossum Schltr., B. reptans (Lindl.) Lindl. ex. Wall., B. striatum (Griff.) Rchb.f., B. sunipia J.J.Verm., Schuit. \& de Vogel, and B. umbellatum Lindl. were collected in the field from the north-eastern region of India representing a broad range of habitats located at varying heights on tree bark and rocky surfaces (Table 1). The plants

Table 1. Details of collection sites and altitude for the twelve species of Bulbophyllum used in the present study

\begin{tabular}{|c|c|c|c|}
\hline $\begin{array}{l}\text { Sl. } \\
\text { No. }\end{array}$ & Species & Collection site & Altitude range $(\mathrm{m})$ \\
\hline 1. & B. affine Wall. ex. Lindl. & Mukhaialong Community Reserve, Jaintia Hills, Meghalaya & $100-1800$ \\
\hline 2. & B. ambrosia (Hance) Schltr. & $\begin{array}{l}\text { Planted at Botanical Survey of India, Eastern Regional } \\
\text { Centre Garden, Shillong (collected from Mizoram) }\end{array}$ & $300-1300$ \\
\hline 3. & B. bisetum Lindl. & Cherapunjee, Khasi Hills, Meghalaya & $1500-2000$ \\
\hline 4. & B. cauliflorum Hook.f. & Chyrmang Community Reserve, Jaintia Hills, Meghalaya & $600-2000$ \\
\hline 5. & $\begin{array}{l}\text { B. cherrapunjeense Barbhuiya } \\
\text { \& D.Verma }\end{array}$ & Cherapunjee, Khasi Hills, Meghalaya & 1460 \\
\hline 6. & B. gymnopus Hook.f. & Chyrmang Community Reserve, Jaintia Hills, Meghalaya & $600-2000$ \\
\hline 7. & $\begin{array}{l}\text { B. leopardinum (Wall.) } \\
\text { Lindl. ex. Wall. }\end{array}$ & Mukhaialong Community Reserve, Jaintia Hills, Meghalaya & $1300-3300$ \\
\hline 8. & B. pteroglossum Schltr. & Upper Shillong, Khasi Hills, Meghalaya & $1000-2500$ \\
\hline 9. & $\begin{array}{l}\text { B. reptans (Lindl.) } \\
\text { Lindl. ex. Wall. }\end{array}$ & Tuber Community Reserve, Jaintia Hills, Meghalaya & $300-1600$ \\
\hline 10. & B. striatum (Griff.) Rchb.f. & Tuber Community Reserve, Jaintia Hills, Meghalaya & $1500-2330$ \\
\hline 11. & $\begin{array}{l}\text { B. sunipia J.J.Verm., Schuit. } \\
\text { \& de Vogel }\end{array}$ & Chyrmang Community Reserve, Jaintia Hills, Meghalaya & $900-2300$ \\
\hline 12. & B. umbellatum Lindl. & Upper Shillong, Khasi Hills, Meghalaya & $1000-2200$ \\
\hline
\end{tabular}


were maintained at the Botanical Survey of India (BSI), Shillong (Meghalaya), for the purpose of further anatomical and morphological investigations. The collected taxa were also matched with the online herbaria on the World Checklist of the Royal Botanic Gardens, Kew (https:// wcsp.science.kew.org/qsearch.do).

\section{Microscopic observations and data acquisition}

Fresh aerial roots from each species were fixed in FAA, i.e., formalin : acetic acid : ethanol $50 \% \mathrm{v} / \mathrm{v}$ $(10: 5: 50)$, and then preserved in $70 \%$ ethanol according to Johansen (1940). Hand-cut transverse sections of fresh as well as FAA preserved roots were taken $1-2 \mathrm{~cm}$ from the base of the root. The sections were stained with $1 \%$ safranin in $70 \%$ alcohol and observed under a compound microscope. The number of cell layers in the velamen and the cortex and their thickness, thickening pattern of the exodermis and the endodermis and their thickness, morphology of tilosomes, number of xylem strands in vascular tissue, root and stele diameter, type of tissue in the stele, the occurrence of crystals and their types were determined. The thickness of the layers in root transverse sections was measured with a calibrated stage micrometer. From one plant per species, three mature roots were sectioned, except for B. affine, B. ambrosia, B. cauliflorum and $B$. striatum, where only one root was taken due to the paucity of root material available. For each root ten measurements were taken from random sections. Photomicrographs were taken on a H600L microscope (Nikon, Tokyo, Japan) fitted with a colour high-definition DS-Fi2 camera.

\section{Data analysis}

Mean values and standard errors of the mean (SE) were calculated for the quantitative characters, and correlation coefficients $(r)$ between selected anatomical character pairs, i.e. velamen thickness and number of xylem strands in the vascular cylinder, velamen thickness and root diameter, root diameter and number of xylem strands in the vascular cylinder, stele diameter and root area, stele diameter and cortex thickness, were calculated using the Microsoft Excel ToolPak.

\section{Results}

The results of qualitative observations and quantitative assessments on the morphology and anatomy of aerial roots of Bulbophyllum are summarized in Tables 2 and 3, and illustrated with photomicrograph evidence in figures 1 and 2, with some data also represented graphically using a bar chart (Fig. 3).

External morphology of the roots: The aerial roots were all grey, greenish-grey, or white in colour with a green apex. Root hairs were absent.

Histological examination of the roots: Transverse sections of the roots were mostly circular in outline and were composed of velamen tissue, exodermis, cortex and endodermis (Fig. 1a). The endodermis was single layered, separating the cortex from the vascular tissue. The diameter of the root and its cross-section area ranged from 0.57 $\pm 0.040 \mathrm{~mm}$ and $0.25 \pm 0.009 \mathrm{~mm}^{2}$ respectively in $B$. striatum to $1.32 \pm 0.051 \mathrm{~mm}$ and $1.37 \pm 0.008 \mathrm{~mm}^{2}$ in B. umbellatum. The roots of half of the examined species viz., B. bisetum, B. cherrapunjeense, $B$. gymnopus, B. leopardinum, B. sunipia and $B$. umbellatum were photosynthetic since the cortical cells had chloroplasts (Fig. 1c, e-g, k, 1).

The thickness of the single outermost layer, the velamen, ranged from $40.70 \pm 1.09 \mu \mathrm{m}$ to 151.70 $\pm 3.44 \mu \mathrm{m}$ (Table 3). This layer represented the smallest proportion of the root diameter in $B$. cherrapunjeense (13.1\%) while largest in B. gymnopus (29.9\%) (Fig. 3; Table 3). The cells of the velamen were commonly columnar, thick walled, stained with safranin (i.e. lignified) and devoid of nucleus and cytoplasmic inclusions, indicating that they were dead (Fig. 2a-h, k, 1).

In all the species studied, an exodermis beneath the velamen layer was single layered with the thickness ranging between $20.72 \pm 0.84$ and $38.48 \pm 0.84 \mu \mathrm{m}$. The thickest exodermis was recorded in B. sunipia 


\begin{tabular}{|c|c|c|c|c|c|c|c|c|c|c|c|c|}
\hline 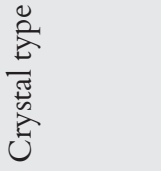 & 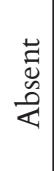 & 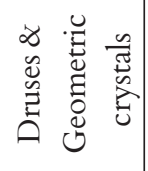 & 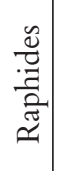 & 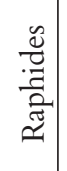 & 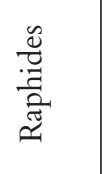 & $\frac{\tilde{v}}{\frac{\tilde{v}}{\pi}}$ & 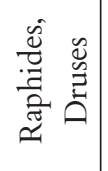 & 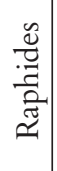 & 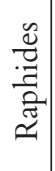 & 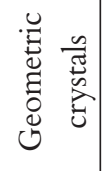 & 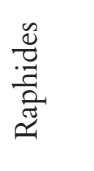 & 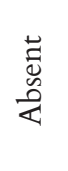 \\
\hline 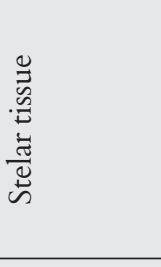 & 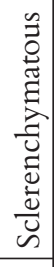 & $\begin{array}{l}\frac{\breve{U}}{0} \\
\frac{\vec{U}}{\tilde{U}} \\
\tilde{n}\end{array}$ & 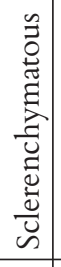 & $\begin{array}{l}\cdot \mathscr{u} \\
0 \\
\dot{U} \\
\tilde{u}\end{array}$ & 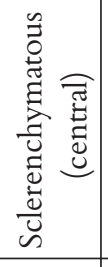 & 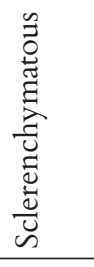 & 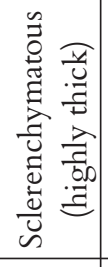 & 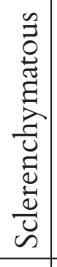 & 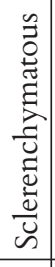 & 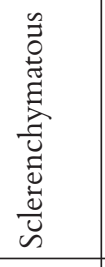 & 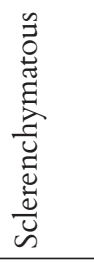 & 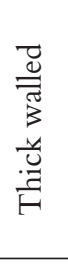 \\
\hline 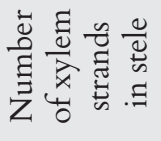 & $\infty$ & $\wedge$ & $\wedge$ & 이 & $\infty$ & $\mathcal{I}$ & $\stackrel{n}{\sim}$ & $\infty$ & $\wedge$ & in & $a$ & \pm \\
\hline 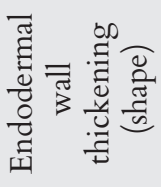 & 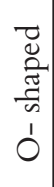 & $\begin{array}{l}\vec{D} \\
\text { בे } \\
\text { ज्ञ } \\
1 \\
0\end{array}$ & 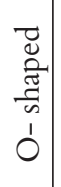 & $\begin{array}{l}\vec{D} \\
0 \\
\frac{2}{7} \\
w \\
1 \\
0\end{array}$ & $\begin{array}{l}\vec{D} \\
\text { בे } \\
\text { ज्ञ } \\
1 \\
1 \\
0\end{array}$ & 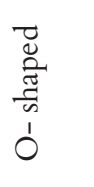 & $\begin{array}{l}\vec{D} \\
\text { בे } \\
\text { ज्ञ } \\
1 \\
0\end{array}$ & 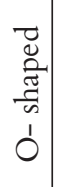 & 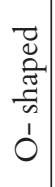 & $\begin{array}{l}\vec{D} \\
\text { בे } \\
\text { ज्ञ } \\
1 \\
0\end{array}$ & 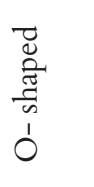 & $\begin{array}{l}\vec{D} \\
\text { D. } \\
\text { ज्ञ } \\
1 \\
0\end{array}$ \\
\hline 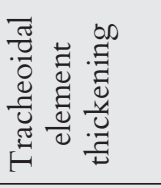 & 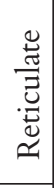 & $\begin{array}{l}\overline{\tilde{n}} \\
\tilde{n}\end{array}$ & $\begin{array}{l}\bar{\pi} \\
\tilde{n}\end{array}$ & $\begin{array}{l}\overline{\tilde{H}} \\
\bar{n}\end{array}$ & 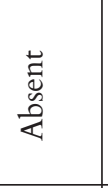 & 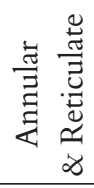 & 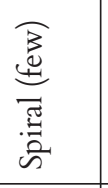 & $\begin{array}{l}\vec{\pi} \\
\vec{n} \\
\tilde{n}\end{array}$ & $\begin{array}{l}\frac{\tilde{c}}{3} \\
\frac{\vec{Z}}{3} \\
\text { 定 }\end{array}$ & $\begin{array}{l}\overline{\tilde{n}} \\
\bar{n}\end{array}$ & 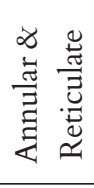 & 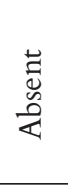 \\
\hline 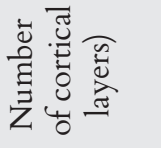 & $\begin{array}{l}\infty \\
1 \\
1\end{array}$ & $\stackrel{I}{I}$ & $\begin{array}{l}10 \\
+\end{array}$ & $\begin{array}{l}n \\
m \\
m\end{array}$ & $\stackrel{n}{+}$ & $\hat{b}$ & $\hat{b}$ & $\begin{array}{c}+ \\
⿱ \\
m\end{array}$ & $\begin{array}{l}10 \\
+ \\
t\end{array}$ & $\begin{array}{l}0 \\
1 \\
1\end{array}$ & $\hat{b}$ & $\begin{array}{l}\infty \\
i \\
i\end{array}$ \\
\hline 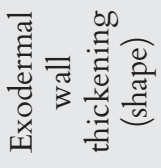 & 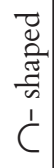 & 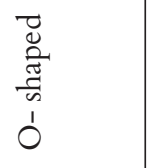 & 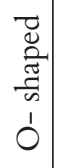 & $\begin{array}{l}\vec{D} \\
\frac{0}{\vec{y}} \\
\frac{\pi}{4} \\
1 \\
0\end{array}$ & $\begin{array}{l}\vec{D} \\
\frac{0}{\vec{z}} \\
\frac{\pi}{n} \\
1 \\
0\end{array}$ & 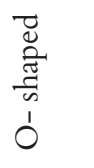 & 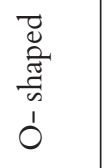 & $\begin{array}{l}\vec{z} \\
\overrightarrow{0} \\
\vec{z} \\
\overline{0} \\
1 \\
0\end{array}$ & 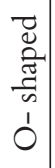 & 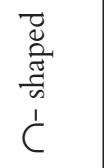 & 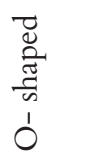 & $\begin{array}{l}\vec{D} \\
0 \\
\text { ज्ञ } \\
\text { n } \\
1 \\
0\end{array}$ \\
\hline 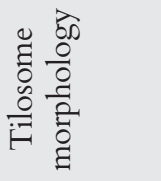 & 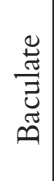 & 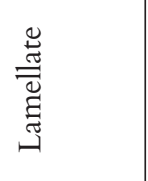 & 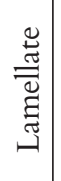 & 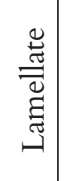 & 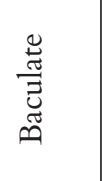 & 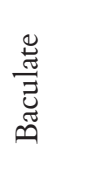 & 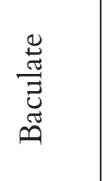 & 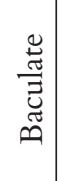 & 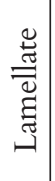 & 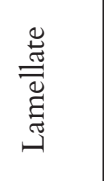 & 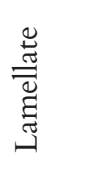 & $\begin{array}{l}\text { के } \\
\text { ह0 } \\
\text { के }\end{array}$ \\
\hline 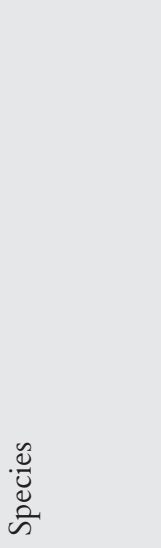 & 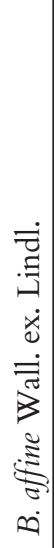 & 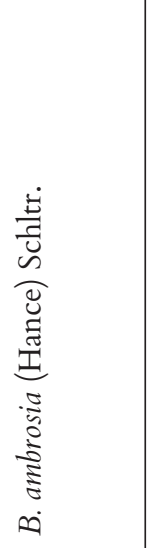 & 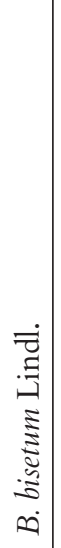 & 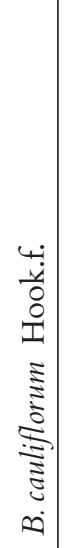 & 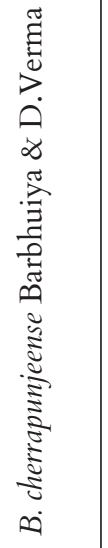 & 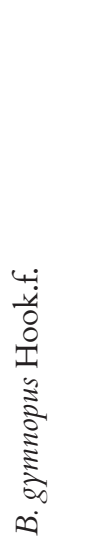 & 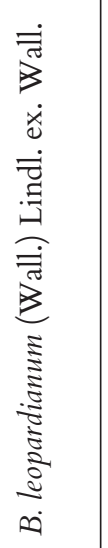 & 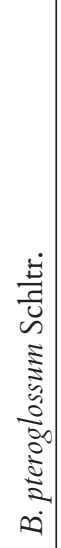 & 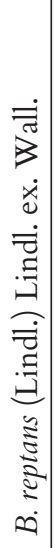 & 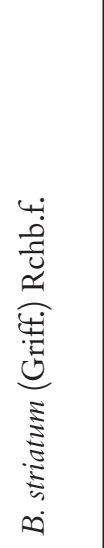 & 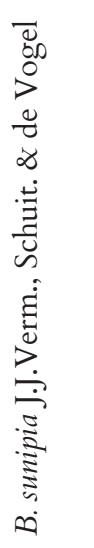 & 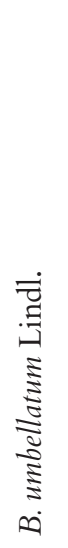 \\
\hline$\dot{\vec{n}} \dot{\mathrm{Z}}$ & -1 & $\sim$ & $m$ & + & in & 0 & $\curvearrowright$ & $\infty$ & $a$ & $\stackrel{ㅇ}{-}$ & $\exists$ & $\stackrel{\sim}{\sim}$ \\
\hline
\end{tabular}




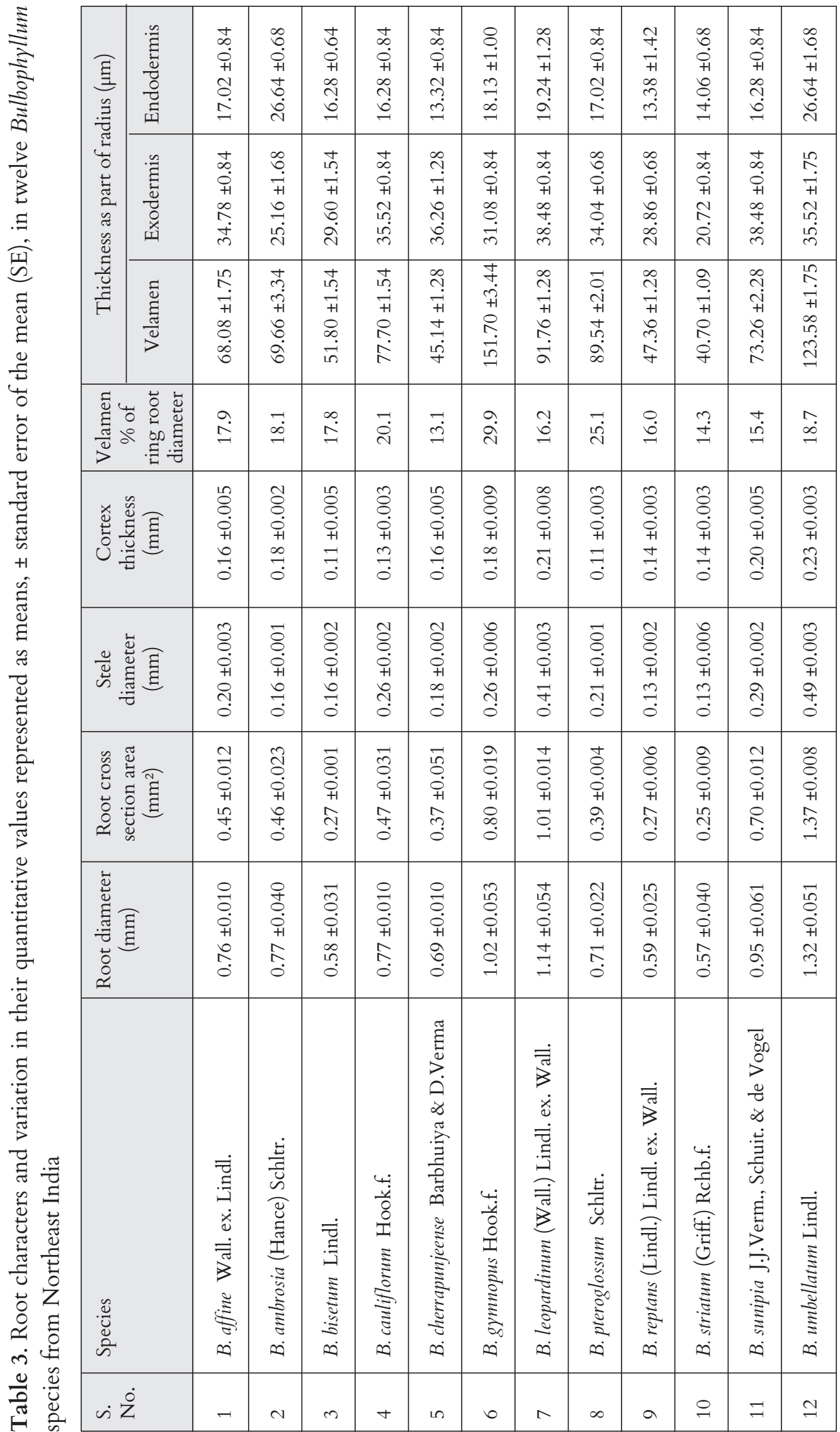


and B. leopardinum while it was thinnest in $B$. striatum (Table 3). The shape of the exodermal cells was polygonal (Fig. 1b-e, g-i, k, 1), or radially elongated (Fig. 1a, f, j). Their cell wall thickening was uniform all around (O-shaped) in the roots of all the species (Fig. 2b-i, k; Table 3), except in $B$. affine and $B$. striatum. In those two species, wall thickening of the outer and radial wall of the exodermal cells was greater compared to the inner walls, thus giving an appearance of an inverted $U$ (i.e., $\cap$-shaped) (Fig. 2a, j). There were a few cells occurring solitary or in pairs in both the exodermal and endodermal layers. Unlike the other cells, they were smaller, thin walled and had a large prominent densely stained nucleus, referred to by Haberlandt (1914) as passage cells (Fig. 2b, f, h, i, j). In the
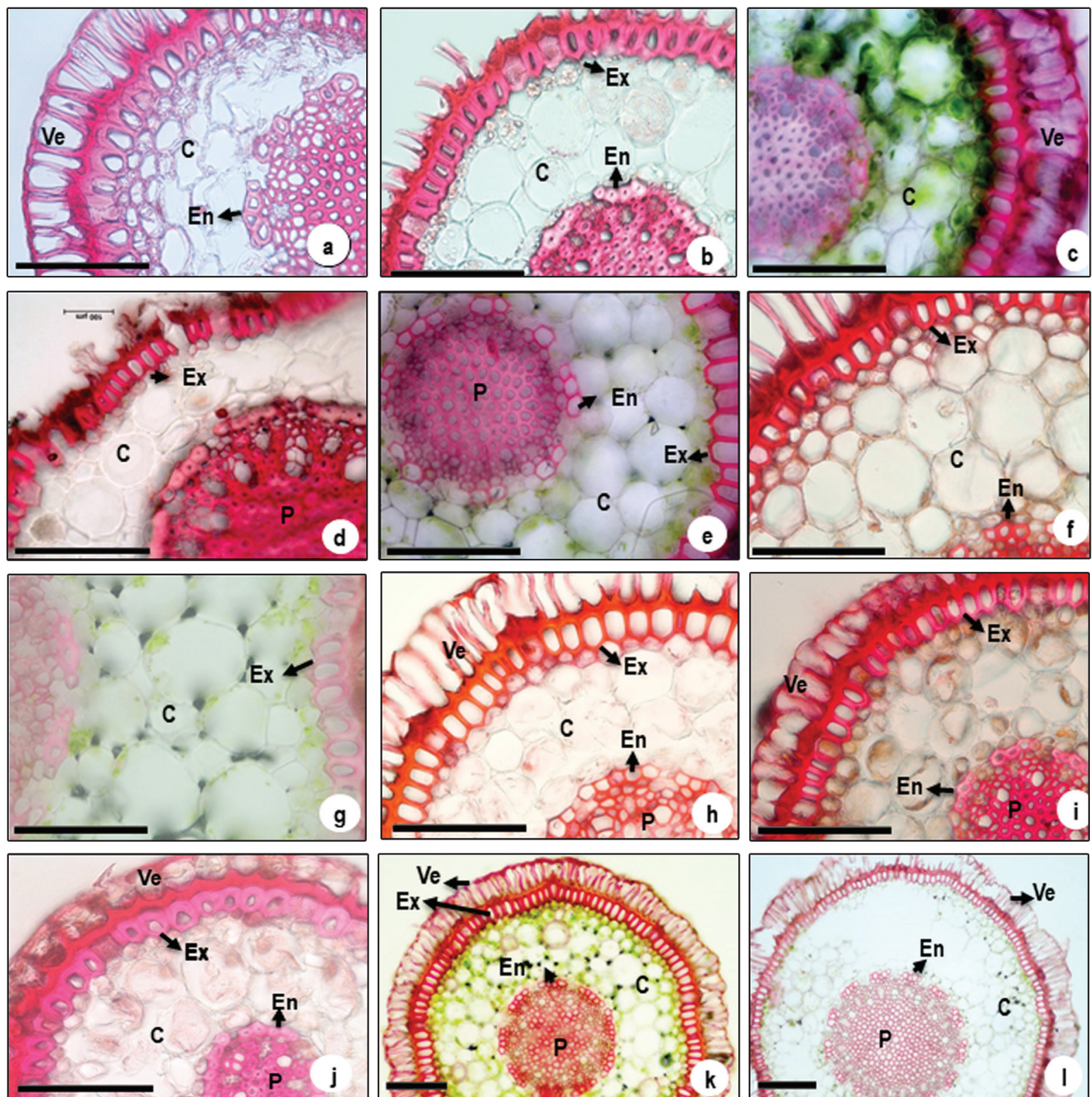

Fig 1. Photomicrographs of root transverse sections of the species of Bulbophyllum Thouars showing layers, viz., velamen, exodermis, endodermis and pith: a. B. affine Wall. ex. Lindl.; b. B. ambrosia (Hance) Schltr.; c. B. bisetum Lindl., chlorenchyma in cortex; d. B. cauliflorum Hook.f.; e. B. cherrapunjeense Barbhuiya \& D.Verma; f. B. gymnopus Hook.f.; g. B. leopardinum (Wall.) Lindl. ex. Wall., chlorenchyma in cortex; h. B. pteroglossum Schltr.; i. B. reptans (Lindl.) Lindl. ex. Wall; j. B. striatum (Griff.) Rchb.f.; k. B. sunipia J.J.Verm., Schuit. \& de Vogel, chlorenchyma in cortex; I. B. umbellatum Lindl., chlorenchyma in the cortex. Abbreviations: $\mathrm{C}=$ cortex; En = endodermis; Ex = exodermis; $\mathrm{P}$ $=$ pith; $V e=$ velamen. Scale bars $\mathrm{a}-\mathrm{j}=100 \mu \mathrm{m}, \mathrm{k} \& \mathrm{I}=200 \mu \mathrm{m}$. 

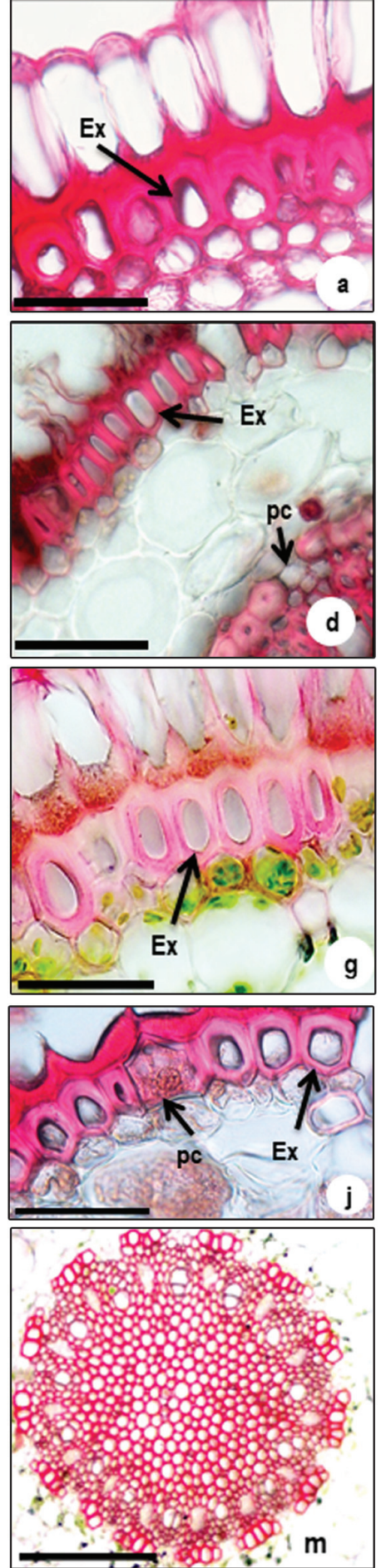
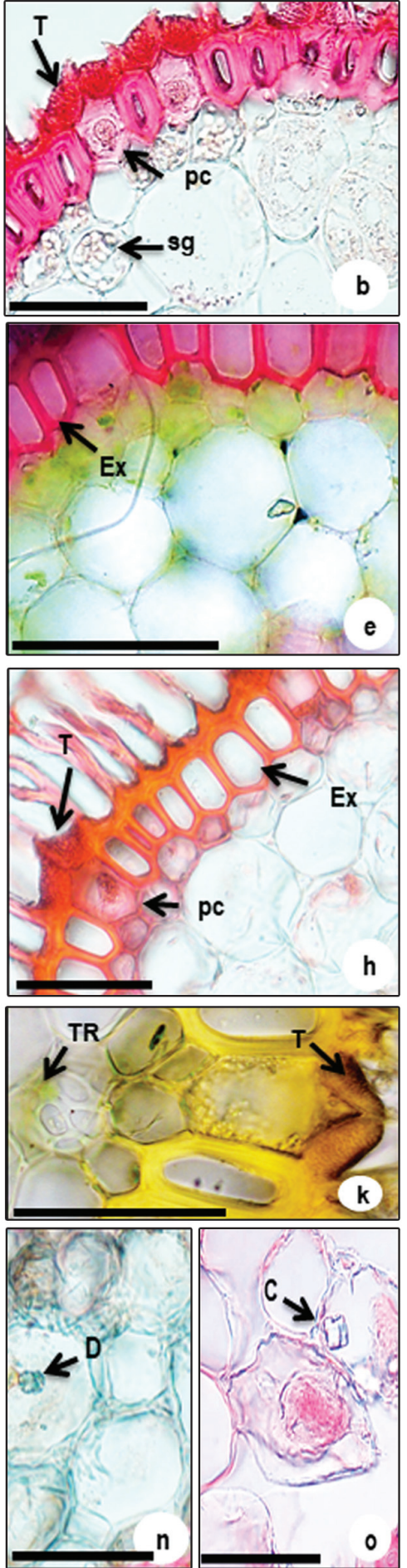
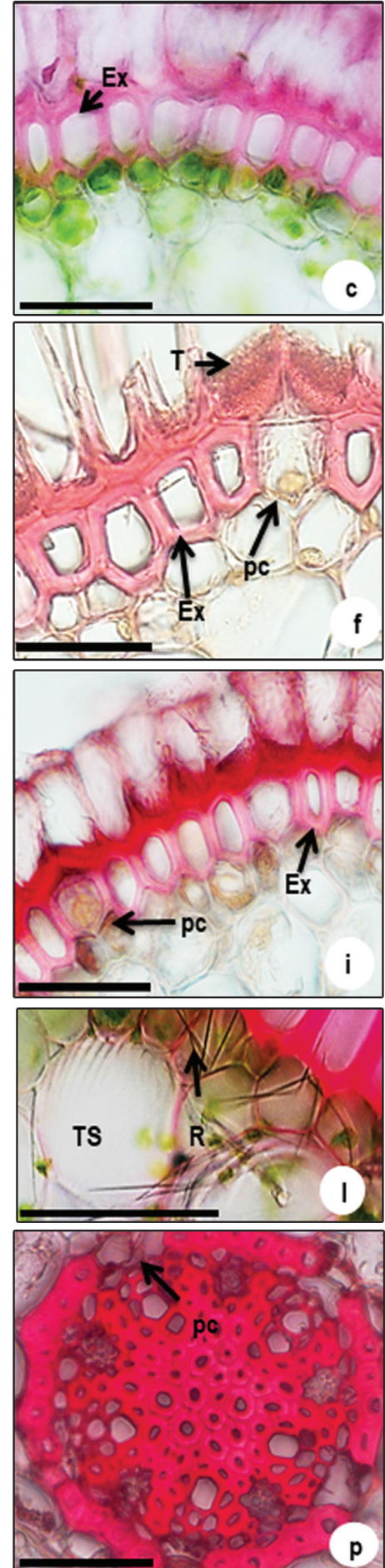

Fig. 2. Photomicrographs of root transverse sections of the species of Bulbophyllum Thouars showing variations in morphological features: a. B. affine Wall. ex Lindl., outer and radial walls of exodermal cells thickened ( $\cap$-shaped); b. B. ambrosia (Hance) Schltr., uniformly thickened walls of exodermal cells, passage cell, starch grains, tilosomes; c. B. bisetum Lindl., exodermal cell wall thickened all over uniformly; d. B. cauliflorum Hook.f., all over uniformly thickened exodermal cell; chlorenchymatous, circular cells in cortex; e. B. cherrapunjeense Barbhuiya \& D.Verma, all over uniformly thickened exodermal cell; f. B. gymnopus Hook.f., all over uniformly thickened exodermal cell, passage cell, tilosomes; g. B. leopardinum (Wall.) Lindl. ex. Wall., all over uniformly thickened exodermal cell; h. B. pteroglossum Schltr., all over uniformly thickened exodermal cell, passage cell, tilosomes; i. B. reptans (Lindl.) Lindl. ex. Wall., all over uniformly thickened exodermal cell, passage cells; j. B. striatum (Griff.) Rchb.f., outer and radial wall thickened ( $\cap$-shaped) exodermal cell, passage cell; k. B. sunipia J.J.Verm., Schuit. \& de Vogel, all over uniformly thickened (0-shaped) exodermal cell, tilosome, tracheoidal idioblast with reticulate thickening; I. B. sunipia J.J.Verm., Schuit. \& de Vogel, tracheoidal idioblasts spirally thickening, raphides; m. B. umbellatum Lindl., passage cell, sclerenchymatous stele; $\mathbf{n}$. B. reptans (Lindl.) Lindl. ex. Wall. Druse in cortex of root transverse section; $\mathbf{0}$. B. striatum (Griff.) Rchb. f., crystal; p. B. striatum (Griff.) Rchb.f. Abbreviations: $\mathrm{C}=$ crystal; $\mathrm{D}=$ druse; $\mathrm{Ex}=$ exodermis; $\mathrm{pc}=$ passage cell; $\mathrm{R}=$ raphide; $\mathrm{sg}=$ starch grain; $\mathrm{T}=$ tilosome; $\mathrm{TR}=$ tracheoidal idioblast with reticulate thickening; TS = tracheoidal idioblast with spiral thickening; ) - inverted 'U'. Scale bars $a-l, n-p=25 \mu m, m=500 \mu m$. 
endodermis, the passage cells occurred precisely opposite the xylem conduits. Fibrous tilosomes were present on tangential walls of the passage cells in the exodermis facing the velamen. In the present investigation, three morphological types of tilosomes, viz., baculate, lamellate and spongy, were observed. The baculate type was observed in $B$. affine, B. cherrapunjeense, B. gymnopus, B. leopardinum and B. pteroglossum (Fig. 2f-h) while lamellate type was most common in half of the species such as $B$. ambrosia, B. bisetum, B. cauliflorum, B. reptans, B. striatum, and B. sunipia (Fig. 2 b-d, f-h, j, k). In contrast, the third type, i.e., spongy type, tilosomes were seen only in B. umbellatum (Table 2).

Across the examined species, the cortex was radially 3-8-celled thick, parenchymatous, in which the cells beneath the exodermis were small polygonal and sclerenchymatous (Fig. 1a, b, f, h, i, k), while in the lower cortex, they were circular to oval and / or polygonal (Fig. 2b, d, e, h). Specialized water storage cells, i.e., tracheoidal idioblasts, were also observed in the cortex region. In all the species, they were circular, polygonal or sac like, with spiral or reticulate thickening, except in B. cherrapunjeense, where they were absent. In the cortex of B. sunipia the water storage cells had both reticulate as well as spiral thickening (Fig. 2k, 1). A preliminary histochemical analysis also showed the presence of calcium oxalate crystals, raphides, and druses (Fig. $21, \mathrm{n}, \mathrm{o}$ ) in cortex cells except in B. affine and B. umbellatum, in which they were absent (Table 2).

The innermost part of root transverse section i.e., stele was circular and sclerotic. The stele of $B$. leopardinum and B. umbellatum proportionally occupied the largest part of the root diameter (36$37 \%$ ) while it was smallest in B. ambrosia (21\%). The cells of the pith were thick walled, lignified and sclerotic in B. cauliflorum and B. ambrosia. The pith region was irregularly sclerenchymatous in $B$. cherrapunjeense while it was uniformly sclerenchymatous in the rest of the species analysed here. Bulbophyllum umbellatum had slightly thick-

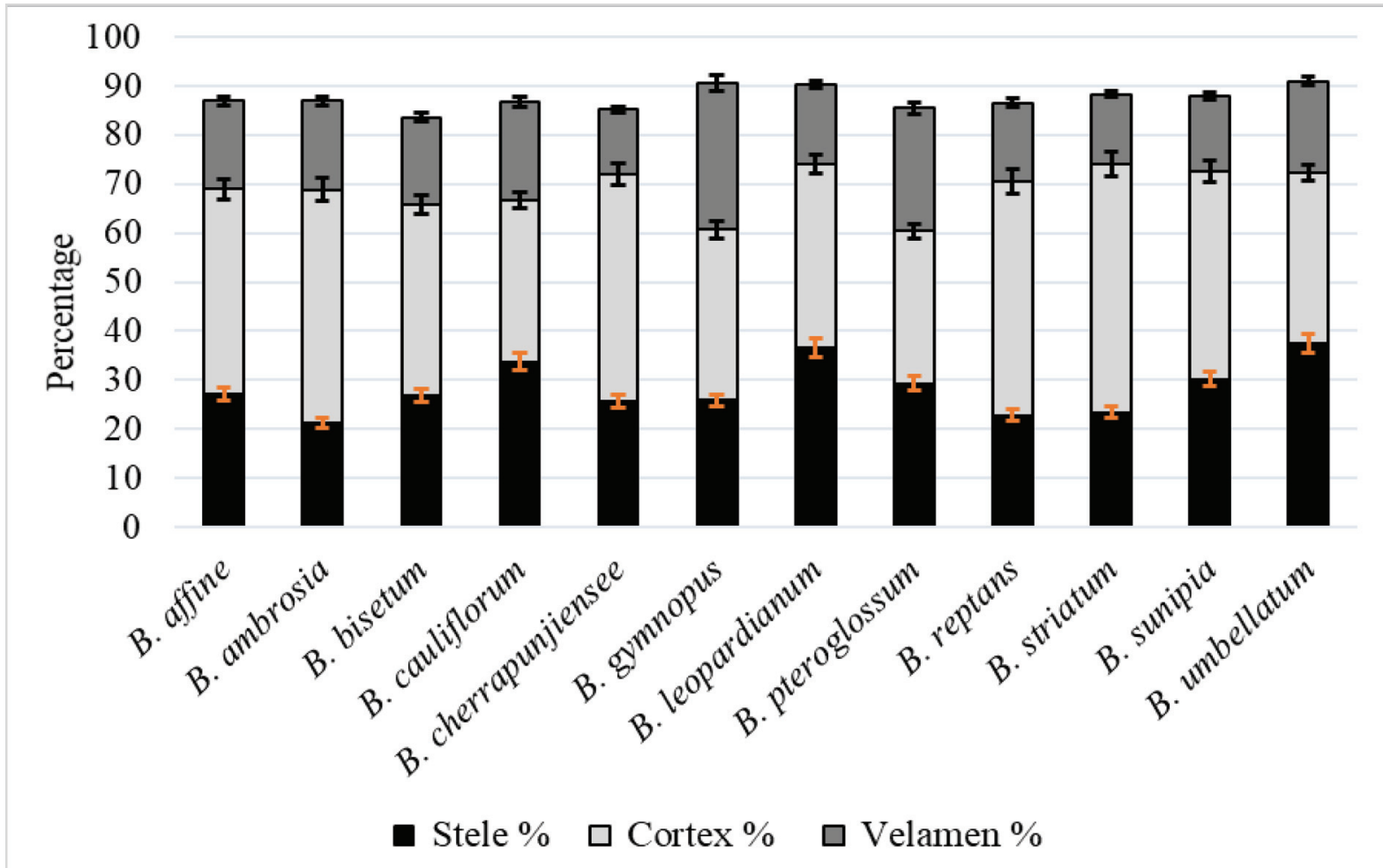

Fig. 3. Percentage of root thickness (diameter) occupied by velamen, cortex and stele in twelve species of Bulbophyllum Thouars from Northeast India. 
walled sclerenchyma (Table 2). The endodermal cells were polygonal, barrel shaped with uniformly thickened walls. They were moderately to highly thick-walled in B. bisetum, B. cherrapunjeense, $B$. gymnopus, B. pteroglossum, B. sunipia and $B$. umbellatum or sclerenchymatous in the remaining species (Fig. 1a-1). The endodermis was uniseriate, thick walled, lignified and separate the cortex from the stele in all the species. These cells were thin walled opposite to all xylem sectors of the vascular cylinder. The stele was polyarch, with xylem and phloem arranged at different radii. The number of xylem strands ranged between 5 and 15 .

Statistically, a positive correlation was found between velamen thickness and number of xylem strands in the vascular cylinder $(r=0.7145, p=$ $0.020243)$, velamen thickness and root diameter $(r$ $=0.7513, p=0.03164$, root diameter and number of xylem strands in the vascular cylinder $(r=0.9198$, $p=0.000164)$, stele diameter and root area $(r=$ $0.9625, p=0.00001)$ and stele diameter and cortex thickness $(r=0.7511, p=0.012296)$.

\section{Discussion}

The root of all species of Bulbophyllum possessed single cell-layered velamen. This outermost layer of dead cells is a specialized epidermis in Orchidaceae (Pridgeon, 1987) and was first described by Oudemans (1861) in Bulbophyllum. This layer was also reported in several other monocot families such as Amaryllidaceae, Araceae, Commelinaceae, Dioscoreaceae and Taccaceae (Dahlgren \& Clifford, 1982). According to an earlier study (Went, 1940), it was believed that the velamen principally absorbs rainwater laden with nutrients that flows down the surface of trees. In contrast, Dycus and Knudson (1957) described that the dead cells of velamen were impermeable to water and provide mechanical support. However, in several later studies it was indicated that the velamen facilitated water absorption (Moreira \& Isaias, 2008; Muthukumar \& Shenbagam, 2018). There are reports that thick-walled exodermal cells are impermeable to water and are said to be the characteristic of orchids growing in drier habitats (Dycus \& Knudson, 1957; Sanford \& Adanlawo, 1973). In several studies the velamen was described as a structure that prevents water loss through the roots (Pridgeon, 1987; Benzing, 1996; Lüttge, 2008).

The cell wall thickening of the exodermis was uniform all around the cell wall (giving an appearance $\mathrm{O}$-shape) in all the species examined here but B. affine and B. striatum showed that the outer and radial walls were thickened ( $\cap$-shaped). Stern (2014) also reported similar results and mentioned the predominance of uniform thickening in the genus Bulbophyllum. The exodermal thickness was found to be moderate to highly thickened in the species examined in present study, as also observed by Porembski and Barthlott (1988). The species like B. affine, B. cauliflorum, B. ambrosia, B. striatum, B. leopardinum and $B$. pteroglossum could be assumed to be better adapted to dry conditions by preventing water loss from interior root tissues as the thick walled exodermal cells are impermeable to water.

According to Haberlandt (1914) the 1-2 distinguishable cells in the exodermis of the aerial roots of orchids are referred to as passage cells. These passage cells may facilitate uptake of water and minerals flowing down along rocks or bark of trees during and after rainfall. Similar passage cells were also recorded in the present study. Presumably such cells may be playing important role in the supply of nutrients to each layer of root. The fibrous tilosomes reported in the present study on the tangential walls of the passage cells (facing the velamen) could possibly enhance the carrier activity of the passage cells.

Three morpho-typic forms of tilosomes were detected in Bulbophyllum species viz., baculate, lamellate and spongy, which were among the seven morphological types of tilosomes identified in orchids as reported by Pridgeon et al. (1983) who also reported their importance as taxonomic marker (Pridgeon, 1987). Scanning electron microscopy details are warranted, which could help to 
substantiate this feature in the investigated species. The available literature indicates that tilosomes act as a water holding structures that help in minimizing the water loss through passage cells (Haberlandt, 1914). It has been shown that tilosomes assist in the condensation of water vapour and other gases such as oxygen and carbon dioxide (Pridgeon, 1987). Figueroa et al. (2008) proposed that the tilosome morphotypes could be indicators of environmental conditions wherein various orchids may be growing and adapted for successful completion of their life cycle. The observations recorded here show two distinct cortex types; either thin with just 3-5 cell layered with various types of thickening pattern and inclusions as a potential storage resource or possessing chloroplast, demonstrating photosynthetic activity. Therefore, histomorphological organization of the cortex could be of some help in species identification. The specialized water storage cells referred to as 'tracheoidal idioblasts' (Foster, 1956) are characteristic to the epiphytic orchids that play an important role in providing mechanical support and protect them from desiccation (Olatunji \& Nengim, 1980; Kaushik, 1983; Porembski \& Barthlott, 1988). Porembski and Barthlott (1988) listed two types of idioblastic cells in the cortex: (i) sac-like cells have different wall thickening patterns and pores, (ii) cells containing raphide-bundles or druses. Tracheoidal idioblasts are absent in $B$. cherrapunjeense, whereas $B$. sunipia showed the presence of reticulate and spiral thickening. Prychid and Rudall (1999) concluded that druses and raphides are the most common types of calcium oxalate crystals in monocotyledons and their presence is also reported by earlier researchers in Orchidaceae (Smith, 1923; Sandoval, 1993; Pridgeon, 1994; Stern, 1997). Druses have been reported in Dendrobium Sw. (Carlsward et al., 1997). Crystals in the roots of Bulbophyllum are of great importance in their occurrence, distribution and type, i.e. druse, raphides and other geometric crystals in the cortex of a particular species (Piazza et al., 2015). The accumulation of such ergastic substances is considered to be genetically controlled (Franceschi
\& Nakata, 2005). Therefore, they may be considered consistent features from a taxonomic point of view.

The endodermis was sclerenchymatous with a considerable variation in the wall thickness. A sclerenchymatous pericycle with presence of passage cells opposite to the xylem strands was considered to facilitate water movement (Pridgeon et al., 2014). The stele was sclerenchymatous in all the Bulbophyllum species studied here.

\section{Conclusion}

The root characters of twelve Bulbophyllum species from Northeast India exhibited several identification features such as the presence of uneven thickening of exodermis cells in B. affine and B. striatum and absence or presence of particular type of crystals in the cortical cells in B. affine and B. umbellatum. The occurrence of raphides in the cortex was found to be a very common feature, whereas geometric crystals were rare and occurred only in B. ambrosia and B. striatum, which could be used as a diagnostic feature. The root anatomical characters viz., tilosome type, crystal type, cell wall thickening patterns described in the present study may be used as species distinguishing features. In the future, scanning electron microscopic (SEM) details of tilosomes might help to explore their taxonomic significance. The interspecific variation in the root morphological characters could assist in the identification of species such as B. gymnopus, $B$. leopardinum, and B. umbellatum. However, the sample of 12 species is at present too small to draw more far-reaching conclusions for the genus until more species are investigated. The application of histochemical techniques will help to develop anatomical tools for the characterization of species.

\section{Acknowledgements}

Authors are grateful to the Head of the Office, Botanical Survey of India, Eastern Regional Centre, Shillong for providing facilities during collection. The expert help of the anonymous reviewers in improving this manuscript is gratefully appreciated. 
This work was partially funded by the CSIR Emeritus Scientist Scheme No. 21(1106)/20/EMRII to SL.

\section{Literature Cited}

ATWOOD J. 1986. The size of the Orchidaceae and the systematic distribution of epiphytic orchids. Selbyana 9: 171"186. https://www.jstor.org/stable/41888801

AYBEKE M. 2012. Comparative anatomy of selected rhizomatous and tuberous taxa of subfamilies Orhidoideae and Epidendroideae (Orchidaceae) as an aid to identification. Plant Systematics and Evolution 298(9): 1643-1658. https://doi.org/10.1007/s00606-0120666-9

AYBEKE M., SEZIKI E. \& G. OLGUN 2010. Vegetative anatomy of some Ophrys and Dactylorhiza (Orchidaceae) taxa in Trakya region of Turkey. Flora. 205: 73-89. https://doi.org/10.1016/j.Flora.2008.11.009

BENZING D.H. 1996. Aerial roots and their environments. In: WAISEL Y., ESHEL A. \& U. KAFKAFI (eds.), Plant roots: the hidden half. Marcel Dekker, New York. pp 875-894.

CARLSWARD B.S., STERN W.L., JUDD W.S. \& T.W. LUCANSKY 1997. Comparative leaf anatomy and systematics in Dendrobium, Section Aporum and Rhizobium (Orchidaceae). International Journal of Plant Science 158(3): 332-343. https://doi.org/10.1086/297445

CHASE M.W., CAMERON K.M., FREUDENSTEIN J.V., PRIDGEON A.M., SALAZAR G., VAN DEN BERG C. \& A. SCHUITEMAN 2015. An updated classification of Orchidaceae. Botanical Journal of the Linnean Society 177 (2): 151-174. https://doi.org/ 10.1111/boj.12234

CHATIN M.A. 1856. Anatomie des plantesaeriennes de I'ordre des orchidees. Premier Memoire: anatomie des racines. Mémoires de la Société Imperiale Académique de Cherbourg 4: 5-18.

DAHLGREN R.M.T. \& D.H.T. CLIFFORD 1982. The monocotyledons: a comparative study. Academic Press, London.

DRESSLER R.L. 1981. The orchids: natural history and classification. Harvard University Press, Cambridge, MA.

DRESSLER R.L. 1993. Phylogeny and classification of Orchid family. Press Syndicate of the University of Cambridge, Cambridge.

DYCUS A.M. \& L. KNUDSON 1957. The role of the velamen of the aerial roots of orchids. Botanical Gazette 119: 78-87. https://doi.org/10.1086/335966

FAN J., He R., JHANG Y. \& X. ZIN 2014. Systematic significance of leaf epidermal features in Holcoglossum
(Orchidaceae). PLoS ONE 9 (7): e101557. https:// doi.org/10.1371/journal.pone.0101557.

FIGUEROA C., SALAZAR G.A., ZAVALETA A.H. \& M.E. ENGLEMAN 2008. Root character evolution and systematics in Cranichidinae, Prescottinae and Spiranthinae (Orchidaceae, Cranichideae) Annals of Botany 101(4): 509-520. https://doi.org/10.1093/aob/ mcm328

FOSTER A.S. 1956. Plant idioblasts: remarkable examples of cell specialization. Protoplasma 46: 184-193. https:// doi.org/10.1007/BF01248877

FRANCESCHI V.R. \& P.A. NAKATA 2005. Calcium oxalate in plants: formation and function. Annual Review of Plant Biology 56: 41-71. https://doi.org/10.1146/ annurev.arplant.56.032604.144106

HABERLANDT G.F.J. 1914. Physiological plant anatomy. (translated from $4^{\text {th }}$ German edition by J.M.F. Drummond). MacMillan and Co., London.

JOHANSEN D.A. 1940. Plant microtechnique. McGraw-Hill Book Company Inc., New York \& London.

KAUSHIK P. 1983. Ecological and anatomical marvels of the Himalayan orchids. Today and Tomorrow's Printers \& Publishers, New Delhi.

LEITGEB H. 1864. Die Luftwurzeln der Orchideen. Denkschriften der Kaiserlichen Akademie der Wissenschaften. Mathematisch Naturwissenschaftliche Klasse 24: 179-222. https://www.zobodat.at/pdf/DAKW_24_2_01790222.pdf

LÜTTGE U. 2008. Physiological ecology of tropical plants. Springer. Berlin. https://link.springer.com/book/10.1007/ 978-3-540-71793-5

MEINECKE T. E.P. 1894. Beiträge zur Anatomie der Luftwurzeln der Orchideen. Flora. 78: 133-203. https:/ /www.zobodat.at/pdf/Flora_78_0133-0203.pdf

MICCO DE V. \& ARONNE G. 2012. Occurrence of morphological and anatomical adaptive traits in young and adult plants of the rare mediterranean cliff species Primula palinuri Petagna. The Scientific World Journal 471814. https://doi.org/10.1100/2012/471814

MOREIRA A.S.F.P. \& R.M. DOS SANTOS ISAIAS 2008. Comparative anatomy of the absorption roots of terrestrial and epiphytic orchids. Brazilian Archives of Biology and Technology an International Journal 51(1): 8393. https://doi.org/10.1590/S1516-89132008000100011

MUTHUKUMAR T. \& M. SHENBAGAM 2018. Vegetative anatomy of the orchid Bulbophyllum sterile (Orchidaceae: Epidendroidae). Lankesteriana 18(1): 1322. http://dx.doi.org/10.15517/lank.v18i1.32701

OLATUNJI O.A. \& R.O. NENGIM 1980. Occurrence and 
distribution of tracheoidal elements in the Orchidaceae. Botanical Journal of the Linnaean Society 80: 357-70. https://doi.org/10.21826/2446-8231201873208

OUDEMANS C.A.J.A. 1861. Ueber den Sitz der Oberhaut bei den Luftwurzeln der Orchideen. Verhandelingen der Koninklijke Akademie van Wetenschappen 9: 1-32.

PIAZZA L.D., SMIDT E. De C. \& C. BONN 2015. Anatomia comparada dos órgãos vegetativos de espécies de Bulbophyllum seção Didactyle (Lindl.) Cogn. e Bulbophyllum seção Xiphizusa Rchb.f. (Orchidaceae). Hoehnea 42(1): 171-183 (in Portuguese). http:// dx.doi.org/10.1590/2236-8906-34/2014

POREMBSKI S. \& W. BARTHLOTT 1988. Velamen radicum micromorphology and classification of Orchidaceae. Nordic Journal of Botany 8(2): 117-37. https://doi.org/10.1111/j.1756-1051.1988.tb00491.x

PRIDGEON A.M. 1987. The velamen and exodermis of orchid roots. In: ARDITTI J. (ed.), Orchid biology. Reviews and perspectives IV. Cornell University Press, Ithaca. pp. 139-192.

PRIDGEON A.M. 1994. Systematic anatomy of Orchidaceae: resources or anachronism. In: PRIDGEON A.M. (ed.), Proceedings of the $14^{\text {th }} \mathrm{W}$ orld Orchid Conference. H.M.S.O., London. pp. 84-91.

PRIDGEON A.M., CRIBB P.J., CHASE M.W. \& F.N. RASMUSSEN 2014. Genera Orchidacearum. Volume 6. Epidendroideae (Part 3): Oxford University Press, Oxford.

PRIDGEON A.M. \& W.L. STERN 1982. Vegetative anatomy of the Myoxanthus (Orchidaceae). Selbyana 7: 55-63. https://www.jstor.org/stable/41759565

PRIDGEON A.M., STERN W.L. \& D.H. BENZING 1983. Tilosomes in roots of Orchidaceae: morphology and systematic occurrence. American Journal of Botany 70: 1365-1377. https://doi.org/10.1002/j.15372197.1983.tb07926.x

PRYCHID C.J. \& P.J. RUDALL 1999. Calcium oxalate crystals in monocotyledons: a review of their structure and systematics. American Journal of Botany 84: 725-739. https://doi.org/10.1006/anbo.1999.0975

SANDOVAL Z.E. 1993. Anatomie foliar de Cuitlauzina pendula. Oriquidea $(M e U x)$ 13:181-190.

SANFORD W.W. \& I. ADANLAWO 1973. Velamen and exodermis characters of West African epiphytic orchids in relation to taxonomic grouping and habitat tolerance. Botanical Journal of the Linnaean Society 66(4): 307-321. https://doi.org/10.1111/j.1095-8339.1973.tb02178.x

SINGH H. 1986. Anatomy of root in some Orchidaceae. Acta Botanica Indica 14: 24-32.

SINGH M., VIMALA Y., LAVANIA S. \& D. VERMA 2020. Leaf epidermal features in relation to taxonomy of some species of Bulbophyllum (Orchidaceae) from Northeast India. Rheedea 30(4): 427-443. https:// dx.doi.org/10.22244/rheedea.2020.30.04.02

SMITH E.L. 1923. The histology of certain orchids with reference to mucilage secretion and crystal formation. Bulletin of the Torrey Botanical Club 50: 1-16. https:// www.jstor.org/stable/2479975

STERN W.L. 1997. Vegetative anatomy of subtribe Orchidinae (Orchidaceae). Botanical Journal of the Linnaean Society 124: 121-136. https://doi.org/10.1111/ j.1095-8339.1997.tb01786.x

STERN W.L. 2014. Anatomy of the monocotyledons Volume X: Orchidaceae. University Press, Oxford. https://doi.org/ 10.1093/acprof:osob1/9780199689071.001.0001

THANGAVELU M. \& A. KOWSALYA 2017. Comparative anatomy of aerial and substrate roots of Acampe praemorsa (Rox.) Blatt. \& McCann. Flora Morphology, Distribution, Functional Ecology of Plants 226: 17-28. https://doi.org/10.1016/j.flora.2016.11.001

WENT F.W. 1940. Soziologie der Epiphyten eines tropischen Regenwaldes. Annales du Jardin Botaniquede Buitenzorg 50: 1-98. 